\title{
An indoor fusion positioning algorithm of Bluetooth and PDR based on particle filter with dynamic adjustment of weights calculation strategy
}

\author{
Lingwu Qian $^{1 *}$, Bingjie Yuan ${ }^{1}$ \\ ${ }^{1}$ Department of Automation, Xiamen University \\ Xiamen, Fujian 361102 - China \\ [e-mail: lingwuqian@foxmail.com] \\ ${ }^{*}$ Corresponding author: Lingwu Qian
}

Received January 12, 2021; revised June 2, 2021; accepted Septebmer 11, 2021; published October 31, 2021

\begin{abstract}
The low cost of Bluetooth technology has led to its wide usage in indoor positioning. However, some inherent shortcomings of Bluetooth technology have limited its further development in indoor positioning, such as the unstable positioning state caused by the fluctuation of Received Signal Strength Indicator (RSSI) and the low transmission frequency accompanied by a poor real-time performance in positioning and tracking moving targets. To address these problems, an indoor fusion positioning algorithm of Bluetooth technology and pedestrian dead reckoning (PDR) based on a particle filter with dynamic adjustment of weights calculation strategy (BPDW) will be proposed. First, an orderly statistical filter (OSF) sorts the RSSI values of a period and then eliminates outliers to obtain relatively stable RSSI values. Next, the Group-based Trilateration algorithm (GTP) enhances positioning accuracy. Finally, the particle filter algorithm with dynamic adjustment of weight calculation strategy fuses the results of Bluetooth positing and PDR to improve the performance of positioning moving targets. To evaluate the performance of BPDW, we compared BPDW with other representative indoor positioning algorithms, including fingerprint positioning, trilateral positioning (TP), multilateral positioning (MP), Kalman filter, and strong tracking filter. The results showed that BPDW has the best positioning performance on static and moving targets in simulation and actual scenes.
\end{abstract}

Keywords: Bluetooth positioning, Orderly statistical filter, Group-based trilateration, Weight adjustment strategy, Particle filter

This work was funded by the project "Design of system integration construction scheme based on functions of each module" (No. XDHT2020169A) and the project of "Development of indoor inspection robot system for substation" (No.XDHT2019501A). 


\section{Introduction}

$\mathbf{W}_{\text {ith the continuous acceleration of urbanization, the size of the urban population has }}$ rapidly expanded. About 80 per cent of our daily activities take place in indoor environments. The accuracy of indoor positioning using the Global Navigation Satellite System (GNSS) is seriously affected due to the occlusion of surrounding buildings. To remedy this, a large number of indoor positioning methods and technologies have been proposed based on Bluetooth, Ultra-Wide Band (UWB) [1, 2], Ultrasonic [3], Infrared [4, 5], Wi-Fi [6, 7], ZigBee [8, 9], and machine vision [10, 11]. However, these methods often have many drawbacks, such as high cost or low accuracy, limiting its extensive application. Among the aforementioned indoor positioning technologies, Bluetooth positioning technology holds many advantages. First, Bluetooth technology is nearly always embedded in smartphones so that no additional devices are needed when positioning individual activities in indoor environments. Besides, smartphones are rich in many other sensors, such as the accelerometer, gyroscopes, and geomagnetic sensors, which can further improve indoor positioning accuracy.

Bluetooth positioning methods comprise two major categories: range-based method [12-14] and range-free method [15-17]. Range-based positioning methods include trilateration (TP), multilateral positioning (MP), among others. In the two-dimensional plane, these methods first measure the distances between the target node and at least three base station nodes, all based on RSSI values, and then establish the positioning equation according to the coordinates of these nodes and corresponding distances. Finally, mathematical optimization methods such as maximum likelihood estimation or the least-squares method obtain the final coordinates of the target node. The main limitation of the RSSI ranging method is that the propagation of Bluetooth signals is a significant influence in complex and dynamic indoor environments. The receiving signal at the end node is usually the superposition of the direct wave signal and the indirect wave signal, resulting in the fluctuation of received signal strength (e.g., RSSI value) [18]. Some unified frameworks of the positioning algorithm based on range $[19,20]$ have come to light in recent years. These algorithms construct the likelihood function, which uses the coordinates of target nodes as latent variables and the ranging measurement between target nodes and base station nodes as the observation value. These algorithms calculate the location of the target nodes using Bayes' theorem. The fingerprint positioning (FP) method is a typical range-free positioning method. FP includes both offline and online stages. In the offline phase, Bluetooth base stations are set up, and reference points determined according to specific rules in a positioning space. At each reference point, the RSSI values from different Bluetooth base stations are gathered; they are then saved as an initial fingerprint. Furthermore, all initial fingerprints are further processed to construct the fingerprint database. For the online phase, the position of the target node is found from the RSSI values gathered from different Bluetooth base stations, and this is done using a pattern matching method. However, the performance of fingerprint localization largely depends on the quality of the fingerprint database [16]. The construction of the fingerprint database is tiresome, especially in large and complex buildings, so the workload of the fingerprint database construction will increase dramatically. Also, to ensure the accuracy and reliability of fingerprint positioning, it is also necessary to regularly repeat the fingerprint collection to keep the fingerprint database updated. Hence, the fingerprint positioning system has poor scalability. Besides, the 
Bluetooth positioning method has another drawback. To reduce the power consumption of Bluetooth, the launch rate of Bluetooth is generally less than ten times per second, which is far lower than the launch rate of other positioning tools, such as Lidar ( 3000 times per second). This feature makes a poor real-time performance of Bluetooth tech in positioning moving targets.

Pedestrian dead reckoning (PDR) is another indoor positioning algorithm that relies solely on the smartphone itself and does not require additional peripheral equipment [21]. Under the condition that the current position is known, PDR can estimate the object's position at the next time interval by estimating the moving direction and distance of the object. In recent years, with the development of micro-electro-mechanical system (MEMS) technology, the size and cost of inertial measurement unit (IMU) sensors have decreased to allow researchers to apply PDR to the positioning and tracking of pedestrians. However, PDR only provides relative position information. Furthermore, the error of positioning caused by the IMU sensors may accumulate as the walking distance increases [22]. Hence, PDR requires other positioning technology to assist in giving a more accurate reading.

Considering the practicability and scalability of the Bluetooth positioning system, we propose a new indoor fusion positioning method: BPDW. Generally speaking, BPDW has the following three contributions. First, to address the fluctuations of RSSI values, referring to the method of Indoor POS [23], BPDW gets relatively stable RSSI values by sorting the RSSI values for a period and then eliminating outliers beyond the upper and lower quartiles. Second, to achieve higher positioning accuracy, BPDW allows as many Bluetooth base stations as possible, within a certain distance, to participate in target positioning through permutations and combinations. Third, by attempting to solve low Bluetooth signal transmission frequency, BPDW fuses the results of PDR and Bluetooth positioning using the particle filter algorithm. More importantly, BPDW can automatically adjust the calculation strategy of particle weights according to the motion state of the target, thereby improving positioning performance. The experiments show that the average absolute error of GTP for static target positioning is $0.5404 \mathrm{~m}$, which is less than the trilateration and multilateral positioning methods. The average fundamental error of BPDW for moving target positioning is $0.84 \mathrm{~m}$ and $0.80 \mathrm{~m}$ in two actual indoor environments, which are 17.6 per cent and 33.3 per cent lower than PDR, respectively.

The paper is summarized as follows. Section 2 introduces the overall structure of BPDW. Section 3 details the Bluetooth ranging model and the preprocessing of the RSSI values through an orderly statistical filter and presents the group-based trilateration algorithm. Section 4 presents integration of the results of Bluetooth positioning and PDR through dynamically adjusting the weight calculation strategy based on the particle filter algorithm. Section 5 describes the experimental results of the proposed method. Finally, in Section 6, the conclusion and perspective are provided.

\section{Structure of BPDW}

This paper proposes an indoor positioning method, BPDW, to improve the real-time positioning accuracy of moving targets. The workflow of BPDW is in Fig. 1. 


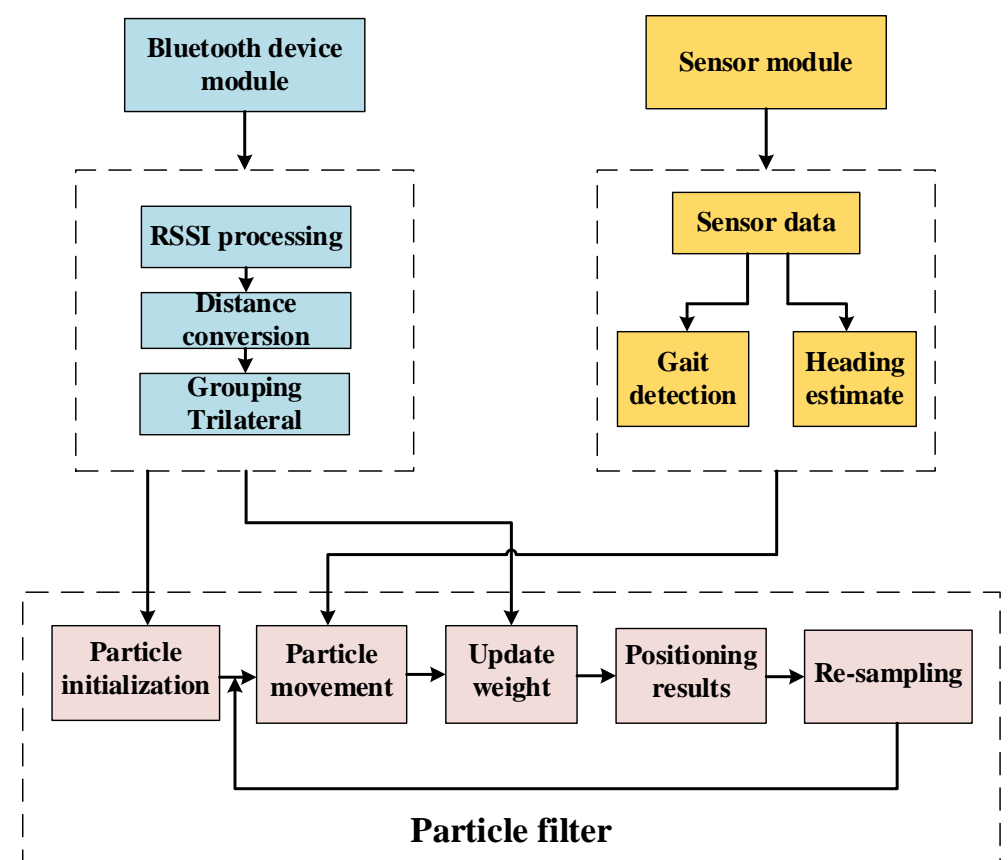

Fig. 1. The workflow of BPDW

Once a target enters the positioning space, BPDW first processes the RSSI values received by the target during a specific period. Next, the orderly statistical filter (OSF) obtains relatively stable RSSI values. Finally, the group-based trilateration positioning (GTP) algorithm positions the target with as many Bluetooth base stations as possible. After that, the position result becomes the initial input of the particle filter. The movement state of the target is determined by the accelerometer embedded in the smartphone. If the target is static, the weight strategy tends to trust Bluetooth positioning more. If the target is moving, the weight strategy tends to trust PDR more, thereby improving the real-time positioning performance for moving targets.

\section{Static Target Positioning}

\subsection{Bluetooth Signal Propagation Model}

The signal strength will reduce gradually when the transmission distance increases in wireless signal propagation. According to the theory of wireless signal propagation [24], in ideal conditions, the spatial propagation model of Bluetooth signals can be expressed as follows:

$$
P_{r}(d)=P_{t} * G_{t} * G_{r} *\left(\frac{\lambda}{4 \pi d}\right)^{n}
$$

where, $P_{r}(d)$ represents the signal power of the receiver when it is $d$ meters away from the transmitter; $P_{t}$ represents the transmitting power of the transmitter; $G_{t}$ and $P_{r}$ represent the antenna gain of the transmitter and the receiver, respectively; $\lambda$ represents the wavelength of Bluetooth signal; and $n$ is the path attenuation factor.

By taking the logarithms of both sides of Equation (1): 


$$
10 \lg \left[P_{r}(d)\right]=10 \lg \left[\mathrm{P}_{t}^{*} \mathrm{G}_{t} * \mathrm{G}_{r} *\left(\frac{\lambda}{4 \pi}\right)^{2}\right]-10 n \lg (\mathrm{d})
$$

Let, $d=d_{0}$, be:

$$
10 \lg \left[P_{r}\left(d_{0}\right)\right]=10 \lg \left[\mathrm{P}_{t}^{*} \mathrm{G}_{t} * \mathrm{G}_{r} *\left(\frac{\lambda}{4 \pi}\right)^{2}\right]-10 n \lg \left(\mathrm{d}_{0}\right)
$$

According to Equation (2) and (3), we get:

$$
P(d)=P\left(d_{0}\right)-10 n \lg \frac{d}{d_{0}}
$$

where,

$$
\left\{\begin{array}{c}
P(d)=10 \lg \left[P_{r}(d)\right] \\
P\left(d_{0}\right)=10 \lg \left[P_{r}\left(d_{0}\right)\right]
\end{array}\right.
$$

Let $P(d)=R S S I(d), P\left(d_{0}\right)=A, d_{0}=1$ then,

$$
R S S I(d)=A-10 n \lg d
$$

According to Equation (6), the distance is:

$$
d=10^{\frac{A-R S S I}{10 n}}
$$

With a fixed parameter $A$ and a known path attenuation factor $n$, the distance between the receiver (target) and the transmitter (Bluetooth base station) is calculated through the RSSI value. Once distances between the target and multiple Bluetooth base station are obtained, the specific location of the target is positioned.

\subsection{Orderly Statistical Filter}

According to the aforementioned Bluetooth signal propagation model, the distance $d$ and the RSSI value possess an exponential relationship. Thus, minor fluctuations of RSSI value cause a vast deviation in the distance. Unfortunately, the RSSI value fluctuates significantly due to the influence of an indoor environment. Therefore, it is necessary to smooth the RSSI values by filtering algorithms.

For outliers not following a regular rule, the classic outlier processing method is used to remove the maximum value and minimum value. If multiple outliers occur within a short time, the method above cannot remove all outliers simultaneously. Therefore, the orderly statistical filter method is adopted to sort the data, which eliminates values above the upper quartile or below the lower quartile and then calculates the mean of the remaining values.

The workflow of the orderly statistical filter is in Fig. 2. First, the RSSI values of the surrounding Bluetooth base stations undergo collection for a specific time. Small squares filled with different colors represent RSSI values from the different Bluetooth base stations. In this period, the RSSI values from the same Bluetooth base station are sorted, and outliers in the RSSI sequence of each Bluetooth base station are discounted. Finally, the remaining RSSI values of sequences are averaged separately.

After using the orderly statistical filter to obtain stable RSSI values, the distances between the Bluetooth base stations and the target node are calculated based on Equation 7, where A and $n$ need to be calibrated according to the collected RSSI values. It is worth noting these values are different in each indoor environment. 


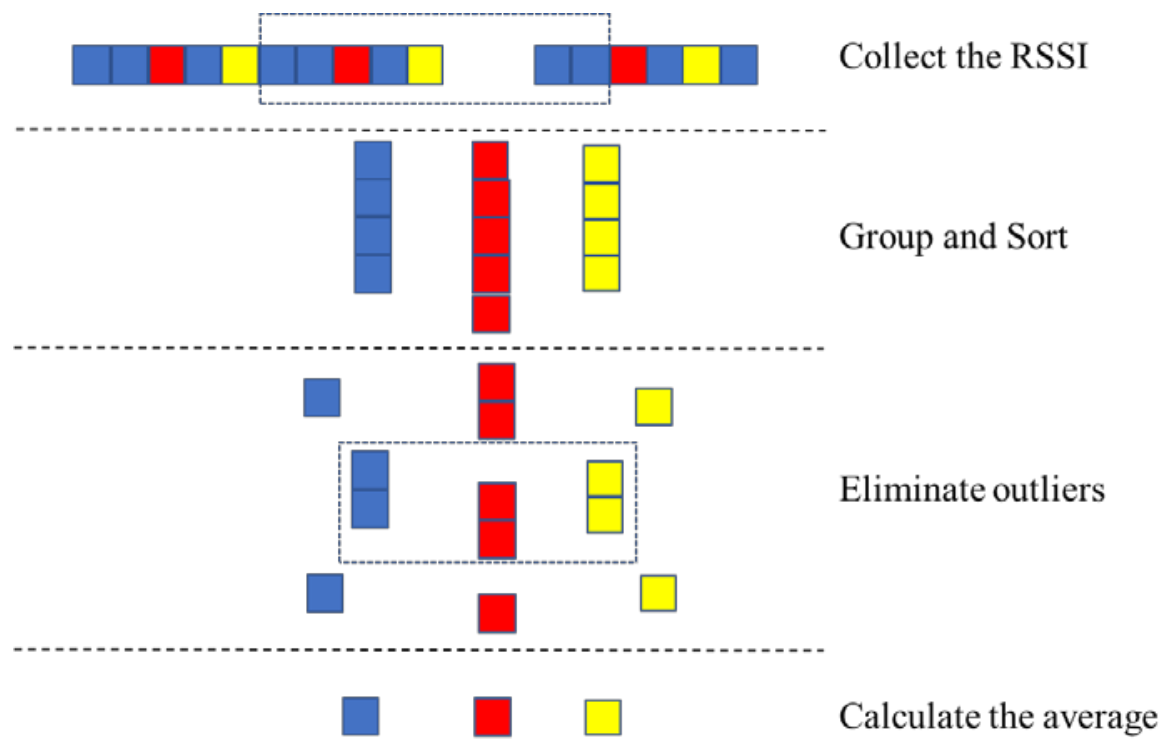

Fig. 2. The process of RSSI processing

\subsection{Group-based Trilateration}

\subsubsection{Trilateration Method}

Among the range-based positioning methods, the trilateration method is a general and straightforward algorithm. If there are three non-collinear anchor nodes, A, B, and C, and an unknown target node $\mathrm{D}$ in the two-dimensional plane, the distances between the three anchor nodes and the target node $D$ measure as $R_{1}, R_{2}$, and $R_{3}$. Three intersecting circles with coordinates of three anchor nodes as the centers and distances $R_{1}, R_{2}$, and $R_{3}$ as their radiuses are intersected at one point. The location of the target node is the intersection point of the three circles, as shown in Fig. 3 (a).

However, due to measurement biases during the actual measurement, the three circles often do not intersect at one point but one area, as shown in Fig. 3 (b). In this case, the position result is solved by many algorithms, such as the maximum likelihood estimation, the least square method, or the triangle centroid algorithm. The procedures of the least square method are as follows [13].

Assuming that the known coordinates of the three anchor nodes are $\left(x_{1}, y_{1}\right),\left(x_{2}, y_{2}\right),\left(x_{3}, y_{3}\right)$, and the unknown coordinates of the target node are $(x, y)$.

(1) Establish the distance equations between Bluetooth base stations and the target node:

$$
\left\{\begin{array}{l}
\left(x_{1}-x\right)^{2}+\left(y_{1}-y\right)^{2}=d_{1}^{2} \\
\left(x_{2}-x\right)^{2}+\left(y_{2}-y\right)^{2}=d_{2}^{2} \\
\left(x_{3}-x\right)^{2}+\left(y_{3}-y\right)^{2}=d_{3}^{2}
\end{array}\right.
$$

(2) Subtract the 3-th equation from the first two equations in the above system of equations, a linearized equation is the result:

$$
A X=b
$$

Where, $A=\left[\begin{array}{ll}2\left(x_{1}-x_{3}\right) & 2\left(y_{1}-y_{3}\right) \\ 2\left(x_{2}-x_{3}\right) & 2\left(y_{2}-y_{3}\right)\end{array}\right], \quad b=\left[\begin{array}{l}x_{1}^{2}-x_{3}^{2}+y_{1}^{2}-y_{3}^{2}+d_{3}^{2}-d_{1}^{2} \\ x_{2}^{2}-x_{3}^{2}+y_{2}^{2}-y_{3}^{2}+d_{3}^{2}-d_{2}^{2}\end{array}\right]$. 
When the inverse matrix of $\mathrm{A}$ exists, there is a unique solution.

$$
\hat{X}=A^{-1} b
$$

Where, $\hat{X}$ is the estimated value of $X$.

(3) Solve by the least square method. Introducing a residual $\varepsilon$, let

$$
\varepsilon_{i}=b_{i}-\hat{b}_{i}
$$

Choose $X$ to minimize the loss function

$$
V_{L S}(X)=\frac{1}{2} \sum_{i=1}^{n-1} \varepsilon_{i}^{2}=\frac{1}{2} \sum_{i=1}^{n-1} \varepsilon^{2}
$$

Find the first derivative of the loss function concerning $X$ and make it equal to 0 , then

Solving this equation:

$$
\left.\frac{\partial V_{L S}(\mathrm{X})}{\partial X}\right|_{X=\tilde{X}}=\frac{1}{2}\left[-2 A^{T} b+2 A^{T} A \tilde{X}\right]=0
$$

$$
\hat{X}=\left(A^{T} A\right)^{-1} A^{T} b
$$

From the knowledge of linear algebra, if the matrix $A^{T} A$ is non-singular, Equation 13 has a unique solution.

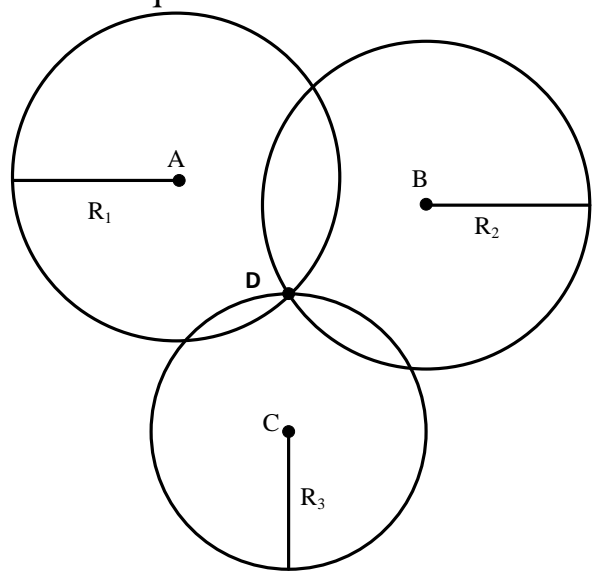

(a) Schematic diagram of the trilateration in the ideal scenario

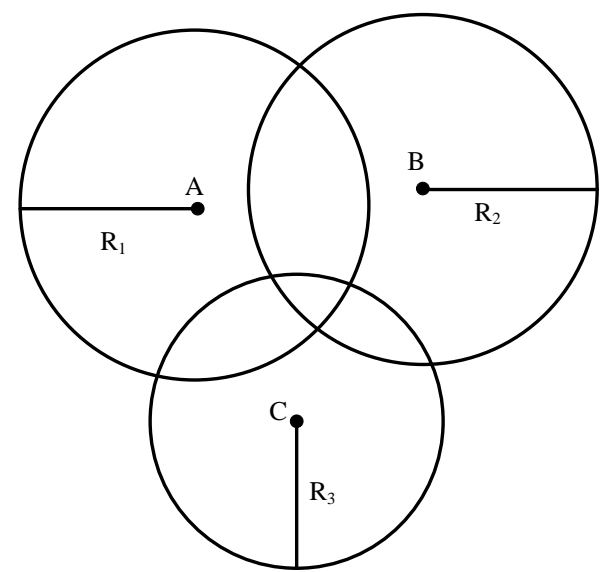

(b) Schematic diagram of the trilateration in the actual scenario

Fig. 3. Schematic diagram of the trilateration

\subsubsection{Group-based Trilateration}

Trilateration generally only uses the RSSI values of three Bluetooth base stations to solve the target position. But under the actual positioning scene, due to the complex indoor environment, some Bluetooth base stations signals may be interfered with and interfered RSSI values can cause a wrong positioning result. Therefore, if more Bluetooth base stations are available and can be used to predict the target location, the positioning accuracy is improved.

After processing RSSI values by the orderly statistical filter, the Bluetooth base stations are grouped in a permutated and combinatorial manner. Then, the Group-based Trilateration (GTP) method uses the trilateration for each Bluetooth base stations group to calculate a positioning result. Next, the calculated results are weighted according to the distance. Finally, the weighted average becomes the positioning result. 
Specifically, when receiving RSSI values from surrounding Bluetooth base stations, the RSSI values are first grouped and sorted according to the Bluetooth base station. The data within the upper and lower quartile range is averaged, and the result is used as the RSSI value of the Bluetooth base station. Then the Bluetooth base stations are sorted according to their RSSI value. If the number of Bluetooth base stations is less than three, this calculation does not occur. However, if there are more than six Bluetooth base stations, only the first six Bluetooth base stations with the maximum RSSI values can solve the target position. Among them, the $\mathrm{k}(\leq 6)$ Bluetooth base stations are grouped into $C_{k}^{3}$ groups. The target location comes from using the abovementioned trilateration method for each group. Based on the principle that the greater the distance the greater the ranging bias, the positioning result of each group will be assigned a weight. The calculation strategy of the final positioning result is as follows.

First, assign an initial weight $\omega_{i}$ to each group as:

$$
\omega_{i}=\frac{1}{d_{i 1}+d_{i 2}+d_{i 3}}
$$

where, $i$ represents the $i$-th group, and $d_{i 1}, d_{i 2}, d_{i 3}$ represents the measured distance from the target to the three Bluetooth base stations in the group. Then normalize the weights as follows:

$$
w_{i}=\frac{\omega_{i}}{\sum_{i=1}^{n} \omega_{i}}
$$

$w_{i}$ is the weight of each group.

The final location of the static target is expressed as:

$$
\left\{\begin{array}{l}
x=\sum_{j=0}^{C_{k}^{3}} x_{i} w_{i} \\
y=\sum_{j=0}^{C_{k}^{3}} y_{i} w_{i}
\end{array}\right.
$$

\section{Moving Target Tracking}

Since the signal launch rate of Bluetooth is usually incredibly low, along with the hysteresis of the orderly statistical filter algorithm, the GTP is poor real-time tracking of the moving target. Taking advantage of the spontaneous nature of PDR, which is not susceptible to external interference, we can effectively make up for the shortcomings of Bluetooth in real-time positioning. Hence, BPDW dynamically adjusts the weight calculation strategy, based on the particle filter algorithm, to integrate the PDR and Bluetooth positioning results. Ultimately, this improves the dynamic performance of tracking moving targets.

\subsection{Particle Initialization}

The first step of the BPDW is to initialize the particles. To ensure that the particles can gather as near as possible to the actual position and improve the positioning accuracy and shorten the time assumption, BPDW first obtains the initial location of the moving target 
$(\hat{x}, \hat{y})$ through the GTP. Simultaneously, the actual state of the system $X$ should be near $(\hat{x}, \hat{y})$, so more particles are placed near $(\hat{x}, \hat{y})$. To meet this sampling requirement, the sampling method used can be expressed as follows:

$$
\left\{\begin{array}{l}
x_{0}^{j}=\hat{x}+d \times \operatorname{rand}() \\
y_{0}^{j}=\hat{y}+d \times \operatorname{rand}()
\end{array}\right.
$$

where $(\hat{x}, \hat{y})$ is the position estimated by the GTP method, $d$ is pedestrian step length, and rand function generates a random value in the range of $[0,1]$.

In this particle initialization stage, the number of sampled particles is $\mathrm{N}$, while the state of each particle represents a possible location of the target. To characterize the similarities between each particle and the actual location of the target, each particle has its own weight information. During the initial stage, i.e., at 0 time instant, the weight of each particle is equal to $1 / \mathrm{N}$. At $\mathrm{k}$-th time instant, the $i$-th sample particle of the system is expressed as:

$$
x_{k}^{i}=\left(x_{k}^{i}, y_{k}^{i}, w_{k}^{i}\right)
$$

where, $x_{k}^{i}, y_{k}^{i}$ is the $\mathrm{x}$-axis and $\mathrm{y}$-axis coordinates of the $i$-th sampled particle respectively, which represents the state of the particle; $w_{k}^{i}$ represents the weight of the $i$-th particle.

\subsection{State Transition}

In BPDW, a system state transition means a gradual change of the target location according to the state transition model. The acceleration sensor and direction sensor built-in for smartphones measure the direction and distance of the movement. The state transition model is as follows:

$$
\begin{aligned}
& x_{k \mid k-1}^{i}=x_{k-1}^{i}+d \cos \alpha \\
& y_{k \mid k-1}^{i}=y_{k-1}^{i}+d \sin \alpha
\end{aligned}
$$

where $d$ is the pedestrian step length, $\alpha$ is the angle between the walking direction and the $\mathrm{x}$-axis of the reference coordinate system.

\subsection{Weight Calculation Strategy}

The weight of the particle characterizes the close degree between each particle and the actual location of the target. The weight of a particle is not only related to the weight of the particle at the preceding moment but also the distance between the current state of the particle and the positioning result of Bluetooth positioning. In BPDW, the smartphone receives a new RSSI value and calculates the target location estimate $\left(\hat{x}_{k}^{\tau}, \hat{y}_{k}^{\tau}\right)$. This fact means that the system obtains a new observation value. According to the theory of particle filter algorithm, the system observation equation is expressed as:

$$
Y_{k}=\left[\begin{array}{l}
\hat{x}_{k}^{\tau} \\
\hat{y}_{k}^{\tau}
\end{array}\right]=\left[\begin{array}{ll}
1 & 0 \\
0 & 1
\end{array}\right]\left[\begin{array}{l}
x_{k} \\
y_{k}
\end{array}\right]+\left[\begin{array}{ll}
\sigma_{k}^{x} & \sigma_{k}^{y}
\end{array}\right]^{T}
$$

Where $\left[\sigma_{k}^{x}, \sigma_{k}^{y}\right]^{T}$ is the observation noise with mean $(0,0)$ and variance $\left(\sigma_{k, x}^{2}, \sigma_{k, x}^{2}\right)$.

The specific calculation process of weight is that when the system detects a target movement and performs particle state transfer, or the system receives a new RSSI value to calculate the target position estimate, it will update the weight of the particle. The weight of each particle is expressed as: 


$$
\begin{aligned}
\overline{\overline{\omega_{k \mid k-1}^{i}}}=p\left(Y_{k} \mid X_{k}^{i}\right) & =p\left(\hat{x}_{k}^{\tau}, \hat{y}_{k}^{\tau} \mid x_{k}, y_{k}\right) \\
& =p\left(\hat{x}_{k}^{\tau} \mid x_{k}\right) p\left(\hat{y}_{k}^{\tau} \mid y_{k}\right) \\
& =\frac{1}{2 \pi \sigma_{k, x}^{2} \sigma_{k, y}^{2}} e^{\frac{\left(\hat{x}_{k}^{\tau}-x_{k}^{i}\right)^{2}}{-2 \sigma_{k, x}^{2}}+\frac{\left(\hat{y}_{k}^{\tau}-y_{k}^{i}\right)^{2}}{-2 \sigma_{k, y}^{2}}}
\end{aligned}
$$

Equation 22 shows that the closer the particle is to the observed location $\left(\hat{x}_{k}^{\tau}, \hat{y}_{k}^{\tau}\right)$, the greater the weight of the particle is.

The weight adjustment strategy of BPDW means that when no target movement is detected, only the Bluetooth signal is received, and the particle weight update calculation still occurs. However, the observed noise variance $\left(\sigma_{k, x}^{2}, \sigma_{k, y}^{2}\right)$ adopts smaller values in Equation 22. On the other hand, when the target movement is detected, the observed noise variance $\left(\sigma_{k, x}^{2}, \sigma_{k, y}^{2}\right)$ uses larger values.

After calculating the weights of each particle, we will update the weights of all the particles and note that the weights of $\mathrm{N}$ particles at k-1-th time instant are $w_{k-1}^{i}$. Thus, the latest weights of $\mathrm{N}$ particles at time $\mathrm{k}$ are expressed as:

$$
\overline{w_{k}^{i}}=\overline{\overline{\omega_{k \mid k-1}^{i}}} \times w_{k-1}^{i}
$$

Then the weights of $\mathrm{N}$ particles $\overline{w_{k}^{i}}$ are normalized to:

$$
w_{k}^{j}=\frac{\overline{w_{k}^{j}}}{\sum_{i=1}^{N} \overline{w_{k}^{i}}}
$$

At k-th time instant, $\mathrm{N}$ particles are obtained from normalization, which can be expressed as $S_{k}=\left\{\left(x_{k}^{j}, y_{k}^{j}, w_{k}^{j}\right), j=1,2, \ldots, n\right\}$. Next, the target location at time $\mathrm{k}$ can be determined by these particles. Generally, two methods are used to determine the estimated position of the final target. One is the maximum posterior criterion, while the other is the weighted average method. To take care of the contribution made by each particle, BPDW uses the weighted-average method to find the estimated location of the target. The estimated location of the target is expressed as:

$$
\left\{\begin{array}{l}
x_{k}=\sum_{j=0}^{N} x_{k}^{j} w_{k}^{j} \\
y_{k}=\sum_{j=0}^{N} y_{k}^{j} w_{k}^{j}
\end{array}\right.
$$

\subsection{Resample}

In BPDW, as the number of iterations increases, the weights of many particles will be smaller, and the weight variance of the particles will continue to increase. This issue will lead to particle degradation problems due to many calculations on particles with a small probability. To remedy this, the particles are resampled. Resampling is to copy particles with larger weight values and eliminate particles with smaller weight values to improve the 
performance of BPDW. Specifically, when the weight of a particle is less than a specific threshold, the particle is removed; the particle with the largest weight is copied at the same time, and random values are added to the position of the copied particle as the position of the new particle. After deleting all the small weight particles, the weights of all particles need to be normalized.

\section{Experimental Results and Discussion}

\subsection{Experimental Setup}

For this paper, experiments took place in an indoor environment on the fourth floor of the Aviation Academy Building at Xiamen University. The Bluetooth was Nordic's NRF52832 chip, supporting 4.2 BLE protocol, a built-in CR2032 button battery holder, a radio transmission power of $0 \mathrm{dBm}$, and a launch rate of $10 \mathrm{~Hz}$. The device to perform the positioning task was an Android smartphone. This is because it has a built-in accelerometer, a direction sensor, a Bluetooth 4.2 chip, and an Android Oreo operating system installed.

\subsection{Bluetooth Signal Propagation Model}

Fig. 4 shows the actual environment of RSSI values collection, measured in the corridor on the 4th floor of the Aviation Academy Building (Fig. 4 (a)). The smartphone and Bluetooth base stations had the same height. The distances to the left and right walls are $1.98 \mathrm{~m}$ (Fig. 4 (b)). For the experiment, in changing the horizontal distance between Bluetooth base station and smartphone, the sampling range was set to 0.5 to $5 \mathrm{~m}$ with a sampling interval of $0.5 \mathrm{~m}$, and collection of 100 data at each sampling point.

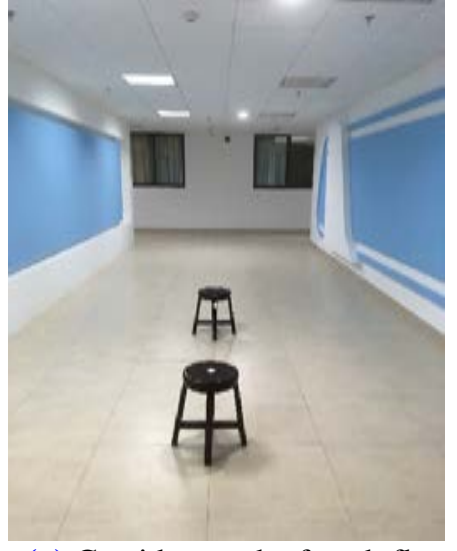

(a) Corridor on the fourth floor

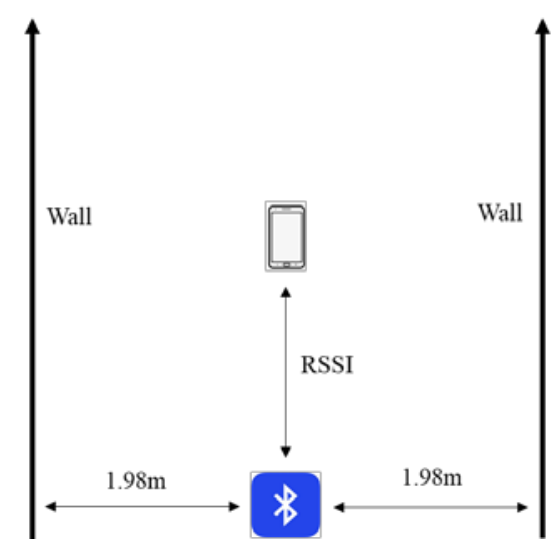

(b) Schematic diagram of collection environment

Fig. 4. RSSI collection environment

Fig. 5 (a) demonstrates the raw data collected by the smartphone at the sampling points of 1 meter, 2 meters, 3 meters, and 4 meters away from the Bluetooth base station. It is evident that the RSSI values of adjacent sampling points partially overlap. Through many measurements, we found that when the sampling distance is greater than 4 meters, as shown in Fig. 5 (b), the RSSI values will overlap, and so the distance measurement based on Equation 7 cannot be performed correctly. Therefore, we recommend that when using Bluetooth base stations, the distance between two adjacent Bluetooth base stations should be within 4 meters. 


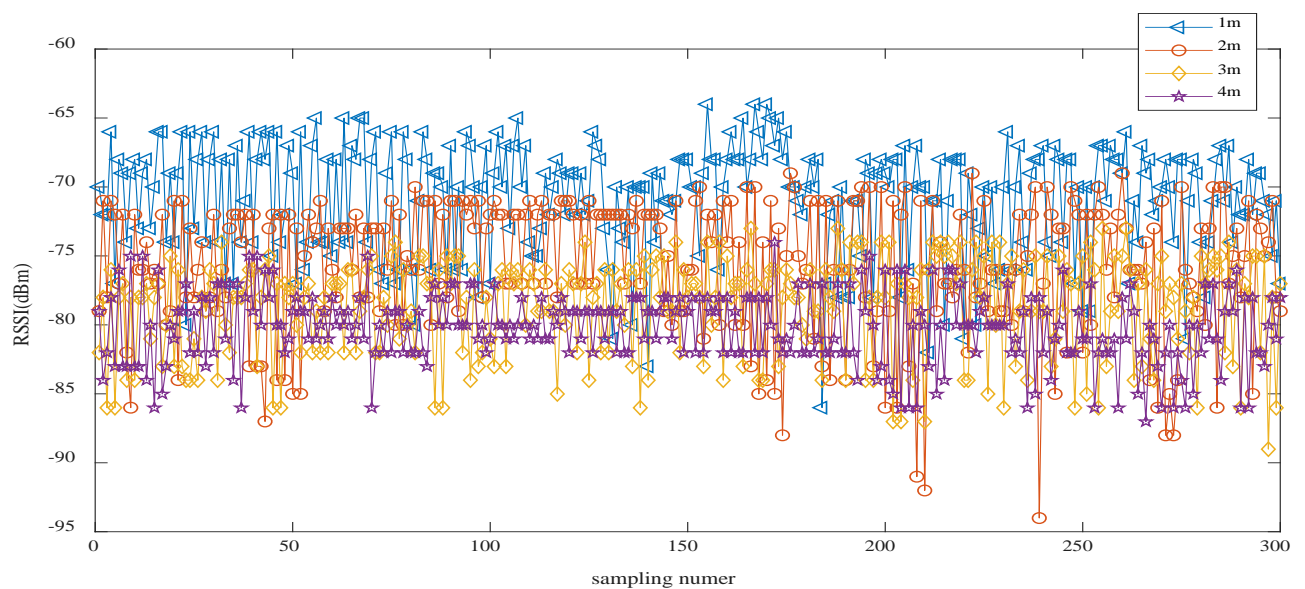

(a) Raw data at sampling points of 1 meter, 2 meters, 3 meters, and 4 meters away from the Bluetooth base station

(b)

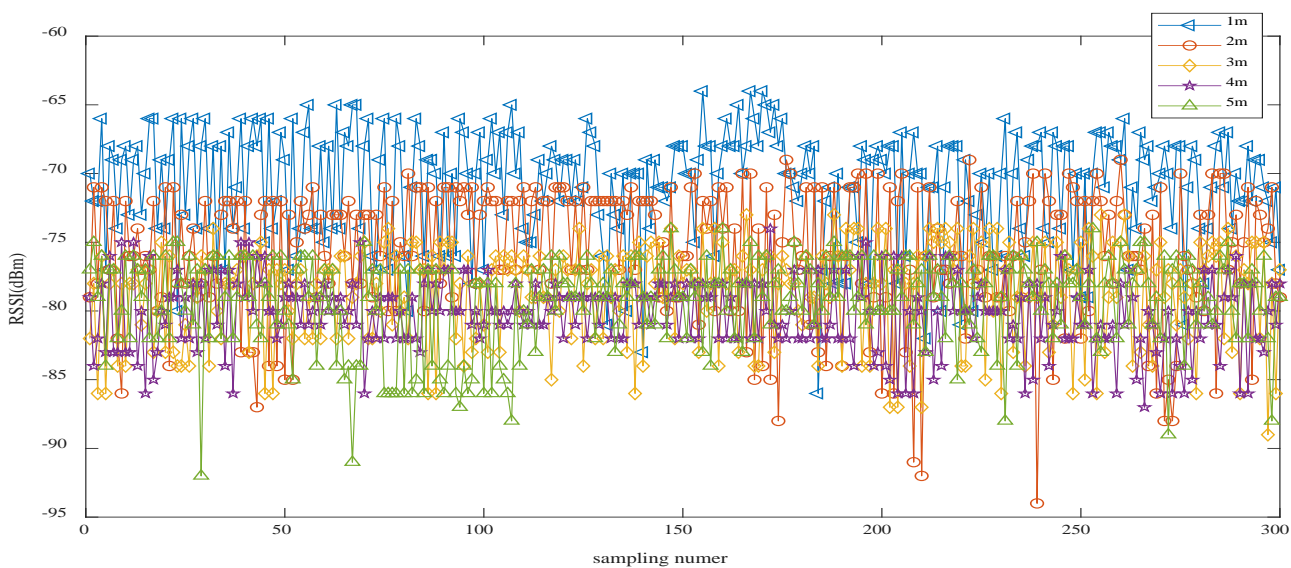

(b) Raw data at sampling points of 1 meter, 2 meters, 3 meters, 4 meters and 5 meters away from the Bluetooth base station

Fig. 5. Raw data collected by the smartphone

We used a total of eight sets of sampled data from 0.5 to 4 meters. Each collection of sampled data contained 100 RSSI values and generated 81 sub-datasets using a sliding window strategy (window size $=20$ and step $=1$ ). Application of the orderly statistical filter on each sub-dataset was used to obtain a relatively stable RSSI value. We used these 648 data points to fit Equation 7 and got $A=-66.21$ and $n=1.891$. Fig. 6 was the fitted curve of the signal propagation model. In all the following experiments, we converted the RSSI value to distance based on this fitted curve. 


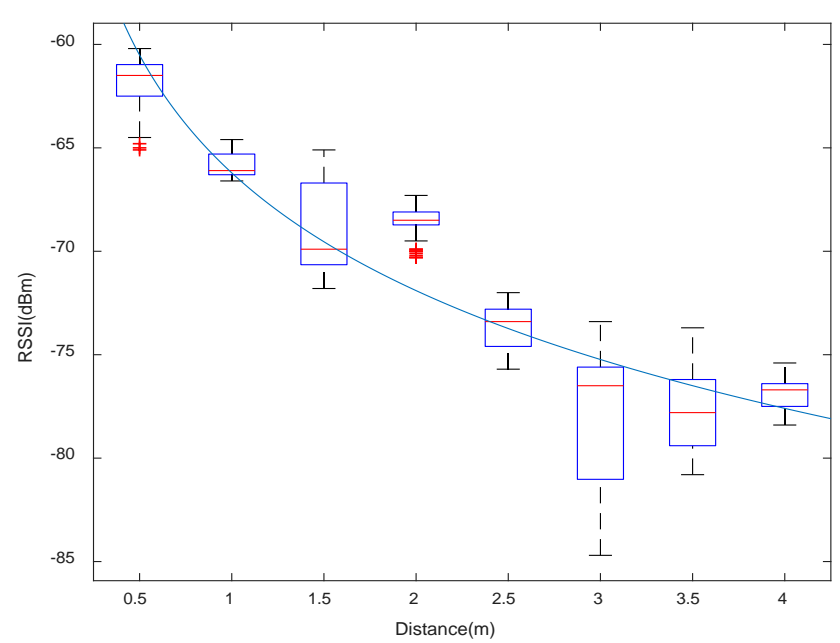

Fig. 6. Bluetooth signal propagation model curve

\subsection{Compared OSF to MMF}

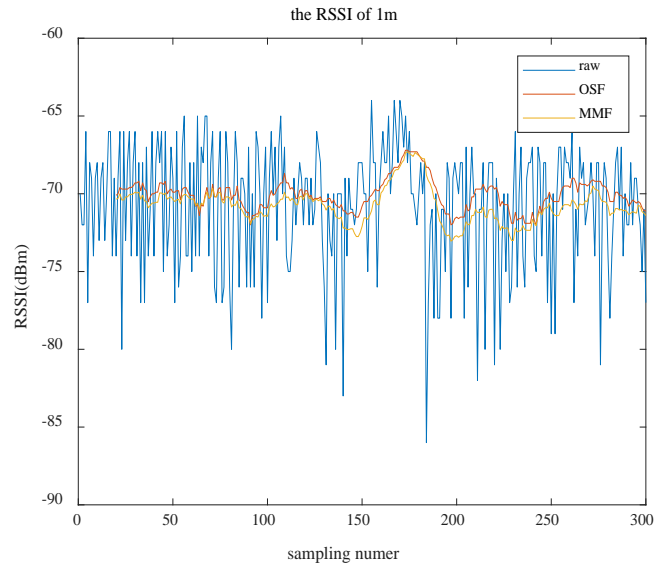

(a) Comparison of filtering results at $1 \mathrm{~m}$

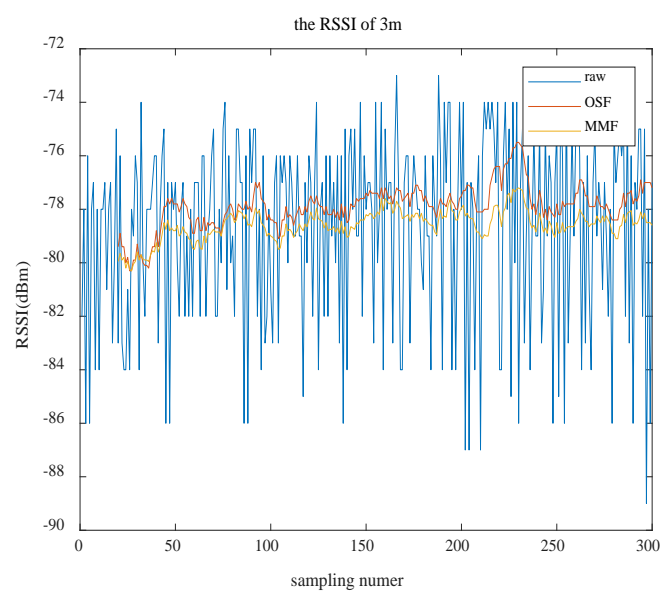

(c) Comparison of filtering results at $3 \mathrm{~m}$

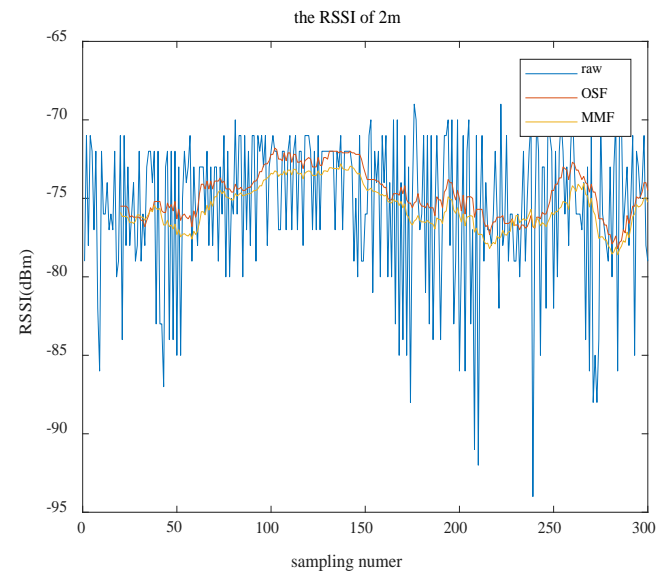

(b) Comparison of filtering results at $2 \mathrm{~m}$

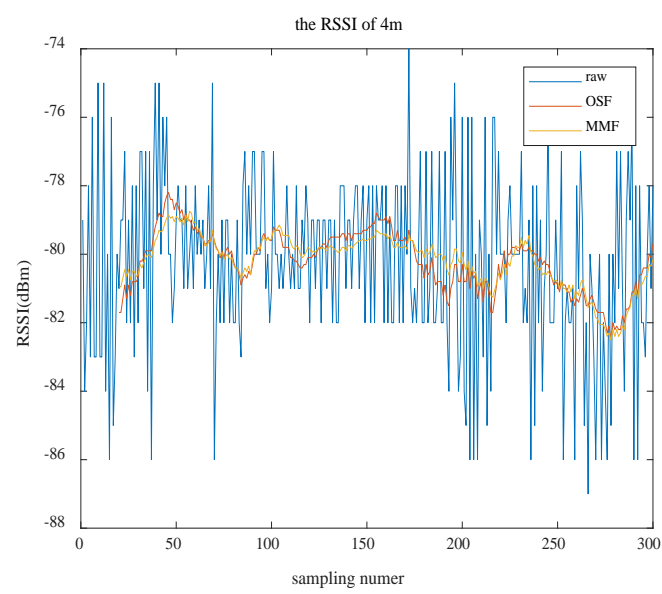

(d) Comparison of filtering results at $4 \mathrm{~m}$

Fig. 7. Comparison of filtering results at different sampling points 
The results of the orderly statistical filter (OSF) and the maximum and minimum filter (MMF) at the four sampling points (1 to 4 meters) are shown in Fig. 7. As seen from the four subplots, the fluctuation ranges of RSSI values are nearly the same, but the value filtering by OSF was larger than the value by MMF. This fact is because OSF eliminates more outliers. Fig. 6 showed that the slight fluctuation of RSSI values may cause a wide error. Therefore, the ranging error caused by OSF will be less than MMF.

\subsection{Comparison Accuracy of Static Target Positioning}

To verify the positioning accuracy of the GTP, we placed the Bluetooth base stations at points of $(0.8,2.4),(4.8,2.4),(4.8,6.4)$, and $(0.8,6.4)$ in an indoor environment. Considering the cost of testing an actual scene, $(2.4,3.2),(1.6,4.0),(2.4,5.6)$, and $(2.4,2.4)$ were evenly selected as the sampling points in the indoor environment. GTP, TP, and MP methods collected 900 valid experimental data at each sampling point, respectively. Finally, a calculation found the average absolute positioning errors between the estimated and actual locations. Note that the three methods all adopt the orderly statistical filter to smooth the RSSI values.

The average absolute positioning errors of the three methods at each sampling point are in Table 1, which demonstrates that the positioning accuracy of the GTP is better than the other two positioning methods. Fig. 8 is a scatterplot of the positioning results at four sampling points. From the four subplots of Fig. 8, we see that the positioning locations of GTP are closer to the targets compared with the other two methods.

Table 1. The average absolute position error

\begin{tabular}{cccc}
\hline \multirow{2}{*}{ Actual coordinate } & \multicolumn{3}{c}{ Average absolute position error (m) } \\
\cline { 2 - 4 } & GTP & TP & MP \\
\hline$(2.4,3.2)$ & 0.4426 & 1.1319 & 1.3672 \\
$(1.6,4.0)$ & 0.5095 & 1.1213 & 0.6454 \\
$(2.4,5.6)$ & 0.2864 & 0.6504 & 0.3756 \\
$(2.4,2.4)$ & 0.8228 & 1.4813 & 1.5590 \\
\hline
\end{tabular}

To further verify the superiority of the GTP method, simulations were done using MATLAB R2017b. In this section, GTP was compared with TP, MP, and fingerprint positioning (FP). This simulation experiment was to arrange Bluetooth base stations every 3 meters in a rectangular area of 6 meters by 6 meters for a total of 9 Bluetooth base stations. For the fingerprint positioning method, a reference point was deployed every meter, creating 49 reference points. At each reference point, 50 RSSI values came from each Bluetooth base station and the averaged RSSI value became the fingerprint of the reference point. At random, 10 locations in the experimental area became targets. Calculation of the positioning errors of 10 targets in each experiment took place, and the experiment was repeated 100 times. The average result became the final positioning error. The results under different noise standard deviations are in Fig. 9. 


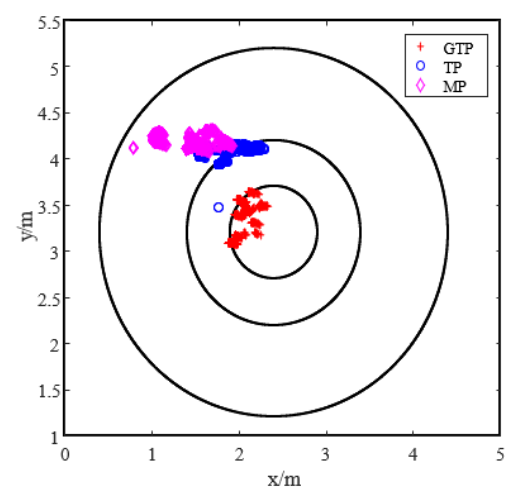

(a) The positioning results scatter plot at $(2.4,3.2)$

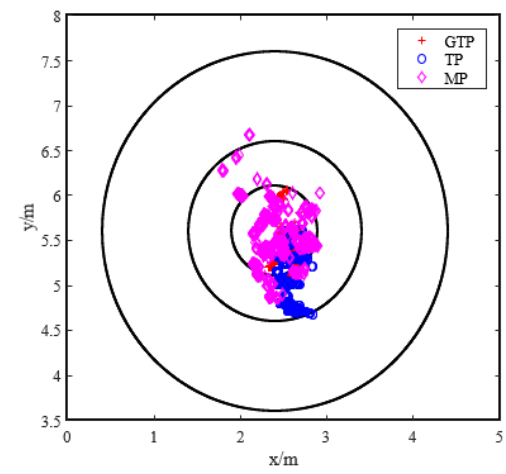

(c) The positioning results scatter plot at $(2.4,5.6)$

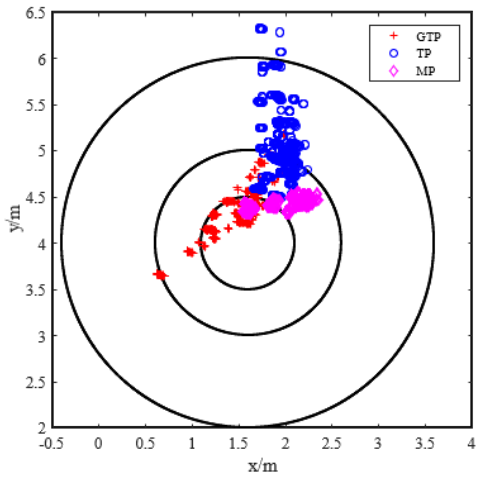

(b) The positioning results scatter plot at $(1.6,4.0)$

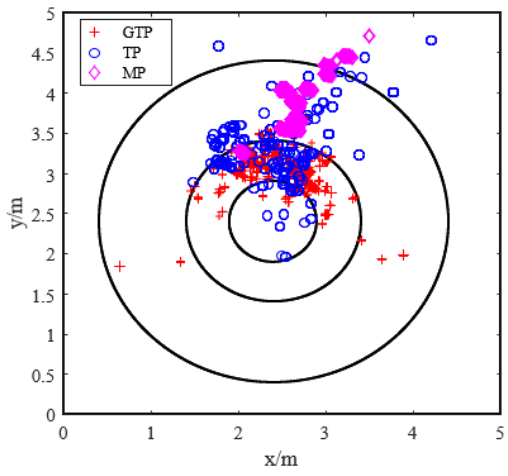

(d) The positioning results scatter plot at $(2.4,2.4)$

Fig. 8. The positioning results' scatterplot at different sampling points

Fig. 9 shows that the result of FP is less affected by noise, but its accuracy is highly related to the construction of the fingerprint database. When the sampling interval of the fingerprint reference points is 1 meter, its positioning error is about 2.5 meter. It is evident from the Bluetooth signal propagation model curve that the closer the Bluetooth base station is to the target, the smaller the ranging error is. Therefore, when the external noise is slight, the positioning accuracy of TP is larger than MP and GTP. However, as the noise continues to increase, the positioning accuracy of TP continues to deteriorate. Simultaneously, due to the use of more information from the Bluetooth base stations, GTP and MP can suppress ranging errors to a certain extent. Compared with MP, GTP can reduce the influence of ranging errors by weighted average. Therefore, even when the noise of the RSSI value was considerable, the performance of GTP was still more efficient than TP and MP. 


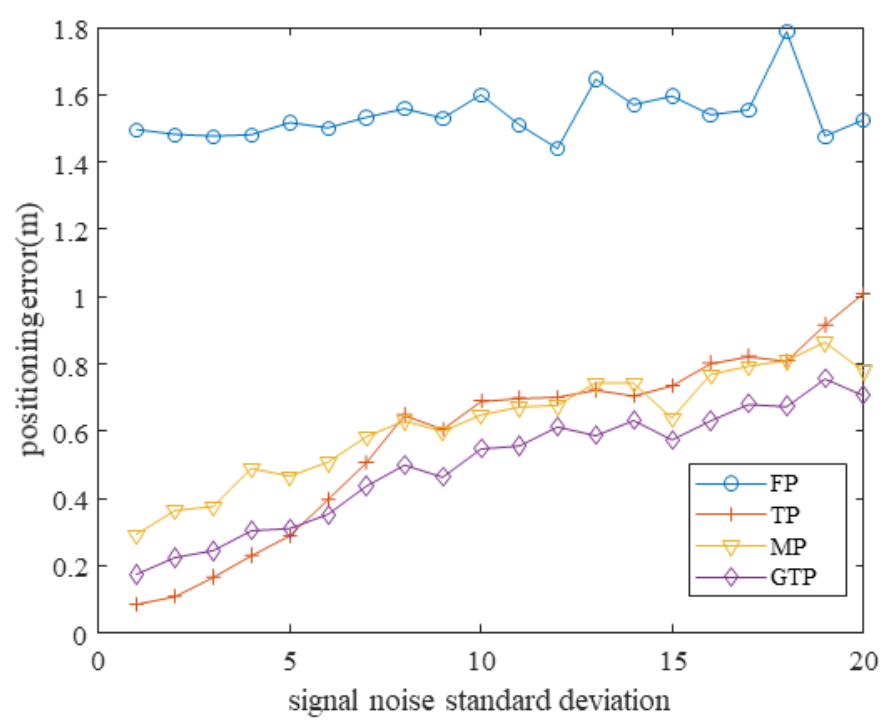

Fig. 9. Comparison results of GTP to TP, MP, and FP

\subsection{Comparison of Moving Target Positioning Accuracy}

To validate the positioning performance of BPDW on moving targets, we first compared it with FP, PDR, and other fusion methods. These included Kalman filter (KF) and strong tracking filter (STF) [25], a comparison of the performance of BPDW and PDR in actual scenarios took place using simulation data. The simulation experiments were performed using MATLAB R2017b.

We simulated the positioning of moving targets. The simulation parameters are as follows: four Bluetooth base stations located at $(0,0),(0,6),(6,0)$, and $(6,6)$, and the moving target shifted along a 6-meter by 6-meter rectangle. Taking into account the number of Bluetooth signals received by the target each step was limited in the actual moving process. During the simulation, the smartphone of pedestrians received only one RSSI value for each step. Besides, the walking model of moving target is as follows:

$$
\begin{aligned}
& L=L_{0}+A+X_{\sigma 1} \\
& \theta=\theta_{0}+B+X_{\sigma 2}
\end{aligned}
$$

where, $L_{0}$ and $\theta_{0}$ are the step length and direction of each step of the moving target. A and $B$ are constants used to simulate the fixed measurement error caused by the sensor, which was set to $A=0.1 \mathrm{~m}, B=3^{\circ} . X_{\sigma 1}$ and $X_{\sigma 2}$ are Gaussian noise with zero mean, where $\sigma 1$ and $\sigma 2$ are standard deviations, was set to $\sigma 1=0.1$ and $\sigma 2=3.87$.

Table 2. Comparison of positioning algorithms $\left(\sigma^{2}=10\right)$

\begin{tabular}{ccccc}
\hline Algorithm & error/m & within $1 \mathrm{~m} / \%$ & within $2 \mathrm{~m} / \%$ & within $3 \mathrm{~m} / \%$ \\
\hline FP & 3.62 & 0 & 4 & 34 \\
GTP & 3.47 & 3 & 5 & 27 \\
PDR & 1.57 & 7 & 92 & 100 \\
KF & 2.78 & 7 & 23 & 54 \\
STF & 2.87 & 6 & 21 & 51 \\
BPDW & 1.43 & 15 & 98 & 100 \\
\hline
\end{tabular}


Table 3. Comparison of positioning algorithms $\left(\sigma^{2}=25\right)$

\begin{tabular}{ccccc}
\hline Algorithm & error $/ \mathrm{m}$ & within $1 \mathrm{~m} / \%$ & within $2 \mathrm{~m} / \%$ & within $3 \mathrm{~m} / \%$ \\
\hline FP & 3.59 & 0 & 4 & 37 \\
GTP & 3.54 & 0 & 6 & 28 \\
PDR & 2.05 & 0 & 43 & 100 \\
KF & 2.94 & 4 & 22 & 51 \\
STF & 2.99 & 3 & 18 & 50 \\
BPDW & 1.91 & 13 & 53 & 100 \\
\hline
\end{tabular}

The simulation results of moving target positioning are in Table 2 and Table 3 . The positioning error of BPDW was reduced by $60 \%, 59 \%, 9 \%, 49 \%$, and $50 \%$ compared with FP, GTP, PDR, KF, and STF, respectively, in the case of a noise variance of 10 . The positioning error of BPDW was reduced by $47 \%, 46 \%, 7 \%$, 35\%, and 36\% compared with FP, GTP, PDR, KF, and STF, respectively, in the case of a noise variance of 25 . From the results of simulation evaluations, as the noise increases, compared with GTP, the positioning error of PDR does not change significantly. This fact is because PDR does not rely on RSSI values for positioning. The positioning performance of BPDW was equivalent to that of PDR, yet slightly better than the PDR method. This occurs because the result of Bluetooth positioning can correct the cumulative errors of PDR to some degree. In contrast, although the Kalman filter and strong tracking filter also fused the results of PDR and Bluetooth positioning, their positioning error was still high. This is because the Kalman filter and strong tracking filter rely heavily on the results of Bluetooth positioning. Therefore, due to the low accuracy of Bluetooth positioning for moving targets, the positioning error of the entire movement is further increased during the movement.

We tested the positioning accuracy of BPDW and PDR in both the corridor and office areas. Three experimenters carried out the experiment, and each repeated the experiment three times. We assumed that each experimenter carried their smartphone and moved along the same route at a constant speed for each test. Finally, a comparison was made between the deviation of the actual path and the positioning result.

Experiment 1 took place in an office area with many obstacles. The entire office area is about 11 meters long and 7 meters wide. For each experiment, the experimenters walked in the shape of a rectangle, enclosed by $(0.4 \mathrm{~m}, 1.6 \mathrm{~m}),(0.4 \mathrm{~m}, 4.6 \mathrm{~m}),(4.0 \mathrm{~m}, 4.6 \mathrm{~m})$, and $(4.0 \mathrm{~m}$, $1.6 \mathrm{~m})$, twice with a constant speed. Finally, the average absolute positioning errors of BPDW and PDR were 0.84 meters and 1.02 meters, respectively. The positioning accuracy of BPDW on moving targets improved by 17.6 per cent compared to PDR. The positioning result is in Fig. 10 (a), and the absolute value of the positioning error is in Fig. 10 (b). In Fig. 10 (a), the solid black line represents the actual walking route, the solid red lines represent the positioning trajectory of BPDW, and the solid blue lines represent the positioning trajectory of PDR. Overall, the red curves are closer to the solid black line than the blue curves. Compared to PDR, BPDW can correct the cumulative errors of PDR in combination with the Bluetooth positioning results. From Fig. 10 (b), at the beginning of BPDW, the positioning errors gradually accumulate. However, when the deviation exceeds a certain value, the error will be corrected. This is because we used the particle filter algorithm, and the PDR positioning result had a greater impact on the particle weight when the target was moving, thus showing the characteristics of PDR. When the error accumulated to a certain extent, there were more invalid particles, and so the particles will be resampled according to the Bluetooth positioning result to correct the deviation of the positioning result. 

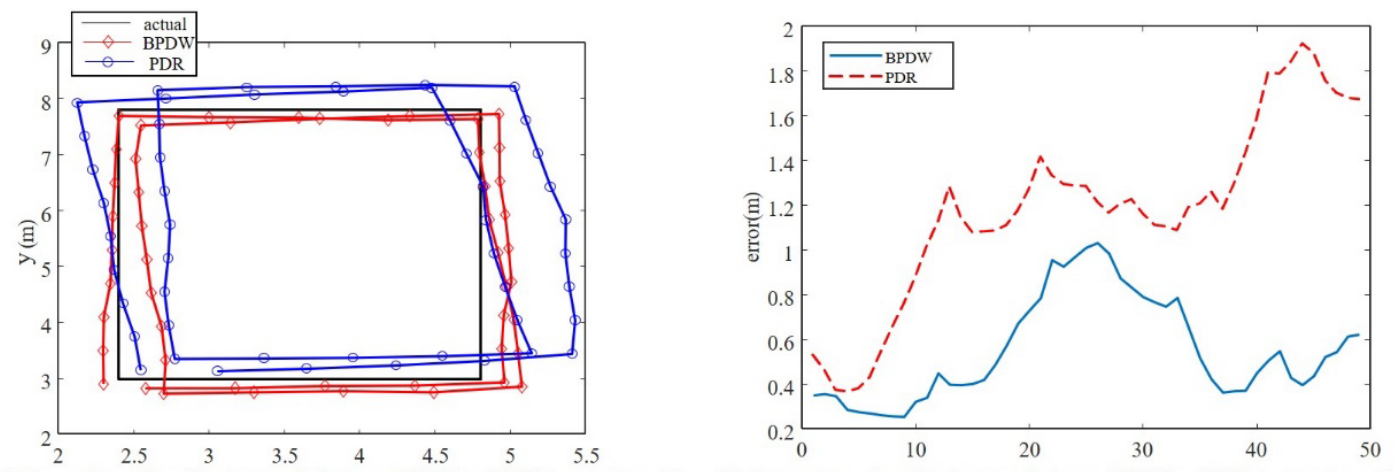

(a) Comparison of positioning results between BPDW and PDR in experiment 1
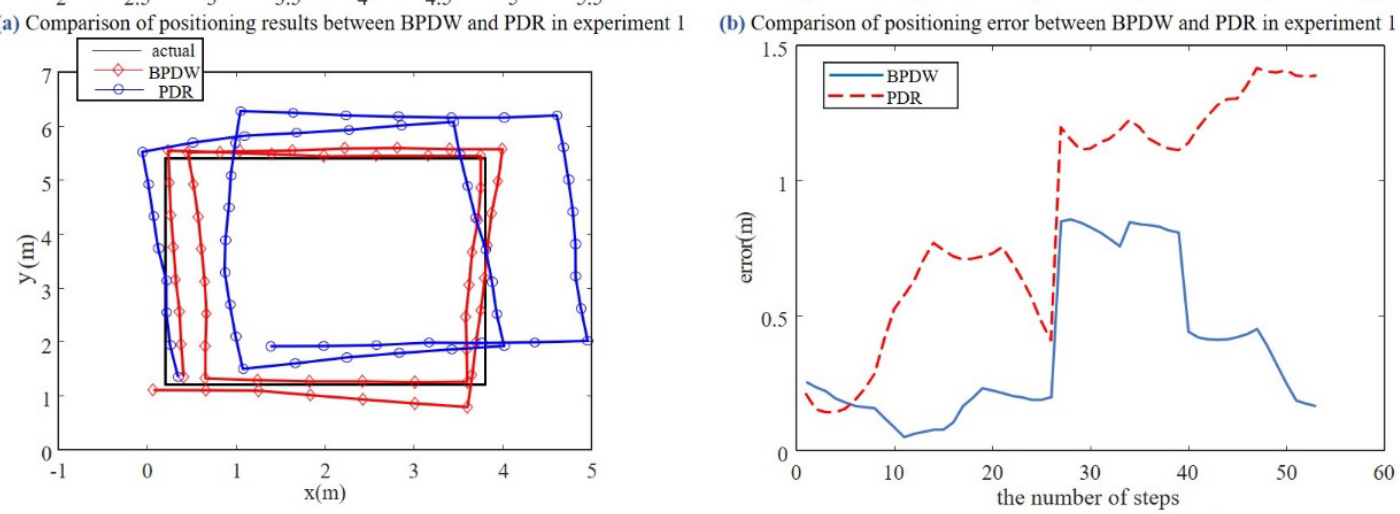

(c) Comparison of positioning results between BPDW and PDR in experiment 2

(d) Comparison of positioning error between BPDW and PDR in experiment 2

Fig. 10. Comparison between BPDW and PDR

Experiment 2 took place in a corridor with few obstacles. The entire corridor is about 11 meters long and 4 meters wide. For each experiment, the experimenters walked a rectangle of 3 meters by 5 meters twice with a constant speed. Finally, the average absolute positioning errors of BPDW and PDR were 0.80 meters and 1.21 meters, respectively, and the positioning accuracy of BPDW for moving targets improved by 33.3 per cent compared to PDR. The positioning result is displayed in Fig. 10 (c), and the absolute value of the positioning error is shown in Fig. 10 (d). Compared with Experiment 1, Experiment 2 increased the total length of the route, so the error of PDR also increased. However, there were fewer obstacles in the corridor environment, so the Bluetooth positioning result was more accurate. Thus, the error generated by PDR was effectively corrected. Therefore, the BPDW positioning effect was better.

\section{Conclusion}

In this paper, we proposed an efficient indoor fusion positioning method based on Bluetooth and PDR. This method can obtain a relatively stable RSSI value by eliminating outliers outside the upper and lower quartiles of the sorted RSSI data. The group-based trilateration integrated with permutated and combinatorial methods can utilize more Bluetooth base stations to improve the performance of Bluetooth positioning. Besides, we combined the positioning results of Bluetooth and PDR based on the particle filter algorithm, which can improve the positioning performance of moving targets significantly. However, the PDR section could use further study on step detection, step length and heading angle 
estimation, improving the positioning accuracy. In addition, more Bluetooth base stations can be used for further testing in a larger range.

\section{References}

[1] T. Janis, S. Florian, and W. Christian, "Design of an UWB indoor-positioning system for UAV navigation in GNSS-denied environments," in Proc. of 2015 International Conference on Indoor Positioning and Indoor Navigation (IPIN), pp. 1-7, 2015. Article (CrossRef Link)

[2] H. Zeng, D. Yang, Y. Wen, J. Qu, and S. Liu, "Design of beam-tilted array antenna for UWB location system," in Proc. of 2015 10th International Conference on Communications and Networking in China (ChinaCom), pp. 100-104, 2016. Article (CrossRef Link)

[3] J. Li, G. Han, C. Zhu, and G. Sun, "An Indoor Ultrasonic Positioning System Based on TOA for Internet of Things," Mobile information systems, vol. 2016, pp. 1-10, 2016.

Article (CrossRef Link)

[4] H. Santo, T. Maekawa, and Y. Matsushita, "Device-free and privacy preserving indoor positioning using infrared retro-reflection imaging," in Proc. of 2017 IEEE International Conference on Pervasive Computing and Communications (PerCom), pp. 141-152, 2017.

Article (CrossRef Link)

[5] H. Yucel, T. Ozkir, R. Edizkan, and A. Yazici, "Development of indoor positioning system with ultrasonic and infrared signals," in Proc. of International Symposium on Innovations in Intelligent Systems and Applications, pp. 1-4, 2012. Article (CrossRef Link)

[6] M. Kotaru, K. Joshi, D. Bharadia, and S. Katti, "SpotFi: Decimeter Level Localization Using WiFi," ACM SIGCOMM Computer Communication Review, vol. 45, no. 4, pp. 269-282, 2015. Article (CrossRef Link)

[7] C. Yang and H. Shao, "WiFi-based indoor positioning," Communications Magazine IEEE, vol. 53, no. 3, pp. 150-157, 2015. Article (CrossRef Link)

[8] X. Yang and Y. Li, "One ZigBee Personnel Location System Based on Fuzzy Logic," in Proc. of 2008 4th International Conference on Wireless Communications, Networking and Mobile Computing, pp. 1-3, 2008. Article (CrossRef Link)

[9] J. Larranaga, L. Muguira, J. M. Lopez-Garde, and J. I. Vazquez, "An environment adaptive ZigBee-based indoor positioning algorithm," in Proc. of 2010 International Conference on Indoor Positioning and Indoor Navigation, pp. 1-8, 2010. Article (CrossRef Link)

[10] A. Xiao, R. Chen, D. Li, Y. Chen, and D. Wu, "An Indoor Positioning System Based on Static Objects in Large Indoor Scenes by Using Smartphone Cameras," Sensors, vol. 18, no. 7, pp. 2229-2235, 2018. Article (CrossRef Link)

[11] W. Liu, Z. Wang, X. Ning, and X. Zhang, "An Object Recognition and Location Based on Binocular Stereo Vision," in Proc. of 2009 International Forum on Computer Science-Technology and Applications, pp. 97-99, 2009. Article (CrossRef Link)

[12] C. Zhou, J. Yuan, H. Liu, and J. Qiu, "Bluetooth Indoor Positioning Based on RSSI and Kalman Filter," Wireless Personal Communications, vol. 96, pp. 4115-4130, 2017. Article (CrossRef Link)

[13] A. E. Waadt, C. Kocks, S. Wang, G. H. Bruck, and P. Jung, "Maximum likelihood localization estimation based on received signal strength," in Proc. of 2010 3rd International Symposium on Applied Sciences in Biomedical and Communication Technologies (ISABEL 2010), pp. 1-5, 2010. Article (CrossRef Link)

[14] S. Wang, B. R. Jackson, S. Rajan, and F. Patenaude, "Received Signal Strength-Based Emitter Geolocation Using an Iterative Maximum Likelihood Approach," in Proc. of Military Communications Conference, pp. 68-72, 2013. Article (CrossRef Link)

[15] Z. Li, L. Xiao, J. Song, C. Gurrin, and Z. Zhu, "A Comprehensive Study of Bluetooth Fingerprinting-Based Algorithms for Localization,” in Proc. of 2013 27th International Conference on Advanced Information Networking and Applications Workshops, pp. 300-305, 2013. Article (CrossRef Link) 
[16] F. Ramsey and H. Robert, "Location Fingerprinting With Bluetooth Low Energy Beacons," IEEE Journal on Selected Areas in Communications, vol. 33, no. 11, pp. 2418-2428, 2015. Article (CrossRef Link)

[17] H. K. Fard, Y. Chen, and K. K. Son, "Indoor positioning of mobile devices with agile iBeacon deployment," in Proc. of 2015 IEEE 28th Canadian Conference on Electrical and Computer Engineering (CCECE), pp. 275-279, 2015. Article (CrossRef Link)

[18] Q. H. Nguyen, P. Johnson, T. T. Nguyen, and M. Randles, "Optimized indoor positioning for static mode smart devices using BLE," in Proc. of IEEE Annual International Symposium on Personal, Indoor, and Mobile Radio Communications, pp. 1-6, 2017. Article (CrossRef Link)

[19] S. Wang, F. Luo, and L. Zhang, "Universal Cooperative Localizer for WSN With Varied Types of Ranging Measurements," IEEE Signal Processing Letters, vol. 24, no. 8, pp. 1223-1227, 2017. Article (CrossRef Link)

[20] W. Yuan, N. Wu, Q. Guo, X. Huang, Y. Li, and L. Hanzo, "TOA-Based Passive Localization Constructed Over Factor Graphs: A Unified Framework," IEEE Transactions on Communications, vol. 67, no. 10, pp. 6952-6965, 2019. Article (CrossRef Link)

[21] W. Kang and Y. Han, "SmartPDR: Smartphone-Based Pedestrian Dead Reckoning for Indoor Localization," IEEE Sensors Journal, vol. 15, no. 5, pp. 2906-2916, 2015. Article (CrossRef Link)

[22] L. V. Nguyen and H. M. La, "A human foot motion localization algorithm using IMU," in Proc. of 2016 American Control Conference (ACC), pp. 4379-4384, 2016. Article (CrossRef Link)

[23] IndoorPos [Online]. Available: https://github.com/megagao/IndoorPos

[24] H. Zhu, M. Li, I. Chlamtac, and B. Prabhakaran, "A survey of quality of service in IEEE 802.11 networks," Wireless Communications IEEE, vol. 11, no. 4, pp. 6-14, 2004. Article (CrossRef Link)

[25] H. Xiong, J. Tang, H. Xu, W. Zhang, W. Zhang, and Z. Du, "A Robust Single GPS Navigation and Positioning Algorithm Based on Strong Tracking Filtering," IEEE Sensors Journal, vol. 18, no. 1, pp. 290-298, 2018. Article (CrossRef Link)

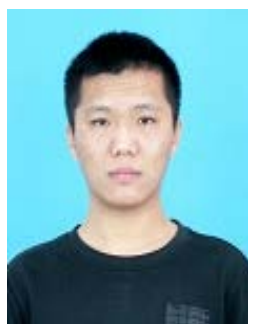

Lingwu Qian was born in China in 1994. He received the B.S. degree from the Department of Automation, Xiamen University of Technology, in 2017. He is currently pursuing the Ph.D. degree in system engineering at Xiamen University. His research interests are in indoor positioning and data processing.

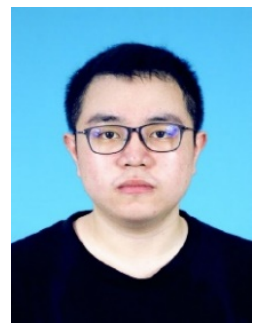

Bingjie Yuan received the B.S. degree in computer science from Fuzhou University, Fuzhou, China, in 2016 and the M.S. degree in software engineering from Fuzhou University \& Yuan Ze University, Fuzhou \& Taiwan, in 2016. He is currently pursuing the Ph.D. degree in system engineering at Xiamen University, Xiamen,China. From 2016 to 2019, his research interest includes the Virtual Reality and Augmented Reality, and his works include image processing. 